Article

\title{
The Prevalence of Metabolic Syndrome and Health-Related Behavior Changes: The Korea National Health Examination Survey
}

\author{
Eunshil Yim ${ }^{1}$, Kyounga Lee ${ }^{2, *(\mathbb{D})}$, Ilsu Park ${ }^{3}$ and Sangjin Lee ${ }^{4}$ \\ 1 Department of Nursing, Daegu Health College, 15 Yeongsong-ro, Buk-gu, Daegu 41453, Korea; \\ yim7604@dhc.ac.kr \\ 2 Medical Research Collaborating Center, Seoul National University Hospital, 101, Daehak-ro, Jongno-gu, \\ Seoul 03080, Korea \\ 3 Department of Healthcare Management, Dong-eui University, 176 Eomgwangno, Busanjin-gu, Busan 47340, \\ Korea; ispark@deu.ac.kr \\ 4 Ministry of Health and Welfare, 13, Doum 4-ro, Sejong-si 30113, Korea; sjlee0709@korea.kr \\ * Correspondence: tj720221@snu.ac.kr
}

Received: 16 April 2020; Accepted: 14 May 2020; Published: 15 May 2020

\begin{abstract}
This study was conducted to investigate the effect of health-related behavior changes on the prevalence of metabolic syndrome (MetS). This study utilized data from the Korea National Health Examination Survey of adults aged 40 or older who underwent health screening in 2011, 2013, and 2015. The prevalence of MetS was analyzed according to sex, age, income, residence location, and health-related behaviors by conducting multiple logistic regression analysis. For health-related behaviors, smoking, drinking, and physical activity were examined, and changes in health-related behaviors over five years from 2011 to 2015 were included in the analysis. The prevalence of MetS in Korea in 2015 was $31.7 \%$. The prevalence showed statistically significant differences according to sex, age, income, location, and health-related behaviors. The prevalence was higher in men than in women and increased with aging. Regarding income, MetS prevalence was slightly higher in the middle-income groups compared with the lowest or the highest. Regarding location, MetS prevalence was lower in metropolitan areas compared to small- to medium-sized cities and farming/fishery rural areas. Regarding health-related behavior, MetS prevalence increased in the smoking, heavy drinking, and passive activity groups compared with the nonsmoking, moderate drinking, and active activity groups. Regarding health-related behavior change, MetS prevalence was higher by $22 \%$ in the short-term nonsmoking group (subjects who smoked in the past but not currently) compared to the continuous nonsmoking group. The risk for MetS also increased by $84.9 \%$ in the continuous heavy drinking group compared to the continuous moderate drinking group. Finally, the risk for MetS increased by $30.3 \%$ in the continuous passive physical activity group compared to the continuous active physical activity group. This study's findings indicate the importance of maintaining healthy lifestyle habits to prevent MetS. In particular, the focus for change should be concentrated on short-term nonsmoking, continuous heavy drinking, and continuous passive physical activities to improve health-related behaviors.
\end{abstract}

Keywords: metabolic syndrome; health behavior; smoking; alcohol intake; physical activity

\section{Introduction}

Metabolic syndrome (MetS) is conceptualized as a cluster of conditions with a high risk for cardiovascular disease and type 2 diabetes, and the presence of MetS is reported to increase the risk for these diseases [1]. The mechanism of the occurrence of MetS is not yet clear, but diverse personal and 
environmental factors (such as smoking, drinking, exercise habits, family history, and education) as well as genetics have been found to be involved [2-6]. According to a meta-analysis study on the relationship between smoking and MetS, there is a significant and positive relationship between active smoking and an increased risk of MetS [2]. Self-reports of moderate consumption of alcohol were associated with a reduction in the prevalence of MetS, serum lipid concentration, and waist circumference compared to those who reported heavy alcohol consumption $[3,4]$. Furthermore, decreased physical activity and an increase in the number of sleeping hours increased MetS prevalence [5]. In another study, the key determinants of MetS were exposure to sedentary lifestyles and obesity [6]. Hence, MetS and cardiac and cerebrovascular disease are believed to be preventable through health-related behaviors such as smoking cessation, moderate drinking, exercise and weight control, and the management of blood pressure, blood sugar, and cholesterol. The National Cholesterol Education Program-Adult Treatment Panel III (NCEP-ATP III) recommends aggressive therapeutic lifestyle changes, including diet, exercise, and education, as a primary strategy in managing patients with MetS $[1,7,8]$.

There has been a significant increase in the prevalence of MetS throughout the world [7,9]. Asian countries have not escaped this trend. In Korea, MetS prevalence in adults has rapidly increased from $24.9 \%$ in 1998 to $31.3 \%$ in 2007, indicating that one out of three adults in Korea has MetS [10]. Currently, seven out of ten primary causes of death in Korea involve chronic degenerative diseases. Of those, cardiac and cerebrovascular diseases have become the primary causes of death, constituting as much as $25.8 \%$ of mortality cases [11]. Accordingly, the National Health Promotion Act was created in Korea for health promotion and disease prevention and the National Health Plan 2020 was established for the promotion of physical activity. A variety of policies were promoted to increase the urgency of improving health-related behaviors and reducing smoking, drinking, and obesity. One of these policies, already implemented, has been conducting health screening and lifestyle habit assessments at least biennially in citizens aged 40 or older to maintain and promote health, minimize economic loss, and reduce health insurance expenditures over a long term. Lifestyle interventions should be conducted at the national level to reduce the burden and consequences of metabolic syndrome [10].

However, it is not easy to modify lifestyle habits that have formed over an extended period of time, and even if a person begins to improve their health-related behaviors, maintaining these new behaviors is not easily done. Among the risk factors for MetS, smoking is a modifiable factor and most smokers attempt to quit smoking. However, less than $5 \%$ of those who attempt smoking cessation succeed in doing so over 12 months, and the success rate for those who reattempt to quit smoking after having previously failed to do so is under $20 \%$ [12].

Health-related behavior change should be maintained over a long period to achieve health benefits because the duration of such change has an impact on the risks for MetS and chronic illnesses [13]. Most previous studies, however, were cross-sectional in design, only investigating the relationship between health-related behavior performance and MetS at the time of study and did not consider the duration of maintaining these behaviors and the pattern of change. A few studies did examine the effects of lifestyle interventions over a six to twelve-month period, but the findings were inconsistent. The researchers pointed out that with a short-term intervention, it may be difficult to see clear effects and that additional research should be conducted to confirm whether the effects of lifestyle modification are retained following the intervention [14-17]. However, it is difficult to maintain healthy behaviors and individuals often revert to unhealthy habits, and therefore the pattern of change should be considered. Thus, the prevalence of MetS should be studied over time regarding health-related behavior changes within a subject and considering the duration of health-related behavior performance. The purposes of this study were to examine the pattern of within-subject changes in smoking, drinking, and physical activity and to identify influencing factors on the prevalence of MetS among health-related behavior changes. 


\section{Materials and Methods}

\subsection{Study Design}

This research was a descriptive and retrospective cohort study aimed at investigating the effects of health-related behavior changes on the prevalence of MetS, using data from the Korea National Health Examination Survey of adults aged 40 or older who underwent general health screening performed by the Korea National Health Insurance Service in 2011, 2013, and 2015.

\subsection{Data Collection and Ethical Considerations}

Upon the approval of the K University Institutional Review Board (IRB No. E1507/001-003), an information disclosure request was made to the Korea National Health Insurance Service. Specifically, data on health screening, insurance eligibility, insurance premiums, and insurance claims were requested. Of the data received from the Korea National Health Insurance Service, those of a total of 578,416 adults aged 40 or older who underwent health screening in 2011, 2013, and 2015 were analyzed in this study.

\subsection{Measuring Health-Related Behavior and Metabolic Syndrome}

\subsubsection{General Characteristics}

Sex was categorized into male and female, and age was grouped into intervals of 10 years. Residence location was classified as metropolitan area, small- to medium-sized city, and farming/fishery rural area. Income level was sorted into quintiles, with a higher quintile ranking indicating a higher income level.

\subsubsection{Health-Related Behavior}

Health-related behavior was defined for each of the items regarding smoking, drinking, and physical activity in the general health screening survey. Smoking was classified into groups of smokers and nonsmokers, which were further defined as current smokers and current nonsmokers including former smokers, respectively, in accordance with the National Health Interview Survey of the US Centers for Disease Control (US CDC). Current smokers referred to those who had smoked over 100 cigarettes during their lifetime and who currently smoke, while nonsmokers referred to those who had never smoked or smoked under 100 cigarettes during their lifetime. Former smokers referred to those who had previously smoked over 100 cigarettes during their lifetime but who do not currently smoke [18]. Drinking was classified into groups of moderate drinkers and heavy drinkers. Heavy drinkers were defined as consuming, on average, more than seven glasses of any alcoholic beverage for men and five glasses for women at least twice per week. All others were classified into the moderate drinking group. These criteria are used in the Korea National Health and Nutritional Examination Survey (KNHANES) in accordance with the World Health Organization (WHO)'s criteria for high-risk alcohol consumption of $60 \mathrm{~g}$ per day for men and $40 \mathrm{~g}$ per day for women [19]. Physical activity was categorized as active and passive, based on the criteria used in the Global Physical Activity Questionnaire (GPAQ) [20]. Being active in physical activity refers to performing one or more activities from high-intensity activity, medium-intensity activity, and walking. The high-intensity activity group consisted of subjects who performed a vigorous workout 30 minutes for more than three times in the past week, and medium-intensity activity group consisted of those who performed a moderately intense workout 30 minutes for more than five times in the past week. The walking group consisted of subjects who walked 30 minutes for more than five times in the last week.

\subsubsection{Health-Related Behavior Change}

Changes in smoking behavior were classified as continuous nonsmoking, short-term nonsmoking, short-term smoking, and continuous smoking. The continuous nonsmoking group consisted of subjects 
who were nonsmokers in 2011, 2013, and 2015, while the continuous smoking group consisted of subjects who were current smokers throughout that period. The short-term nonsmoking group included subjects classified as nonsmokers in 2015 but as current smokers in 2011 and 2013. The short-term smoking group included subjects classified as current smokers in 2015 but as nonsmokers in 2011 and 2013.

Changes in drinking behavior were classified as continuous moderate drinking, short-term moderate drinking, short-term heavy drinking, and continuous heavy drinking. The continuous moderate drinking group consisted of subjects classified as moderate drinkers in 2011, 2013, and 2015 , while the continuous heavy drinking group consisted of subjects classified as heavy drinkers throughout. The short-term moderate drinking group consisted of subjects classified as moderate drinkers in 2015 but as heavy drinkers in 2011 and 2013. The short-term heavy drinking group consisted of subjects classified as heavy drinkers in 2015 but as moderate drinkers in 2011 and 2013.

Changes in physical activity were categorized as continuous passive, short-term passive, short-term active, and continuous active. The continuous passive group consisted of subjects classified as passive in their physical activity in 2011,2013, and 2015, while the continuous active group consisted of subjects classified as active throughout. The short-term passive group consisted of subjects classified as passive in 2015 but as active in 2011 and 2013, and the short-term active group consisted of subjects classified as active in 2015 but as passive in 2011 and 2013.

\subsubsection{Metabolic Syndrome}

The diagnosis of MetS was based on the modified ATP III criteria 13 presented by the American Heart Association (AHA) and the US National Heart, Lung, and Blood Institute (NHLBI), in which racial differences are considered [21]. Considering the difference between Asians and Westerners in the distribution of waist circumference, this study used the Korean Society for the Study of Obesity (KOSSO) criteria for abdominal obesity in the Korean population, i.e., $90 \mathrm{~cm}$ for men and $85 \mathrm{~cm}$ for women [22]. Subjects who met three of the five items of abdominal obesity as determined by waist circumference, hypertriglyceridemia, low HDL cholesterol, high blood pressure, and high fasting glucose were diagnosed for MetS.

\subsection{Statistical Analysis}

Differences in MetS prevalence by sex, age, income, location, and health-related behaviors were analyzed by $\chi^{2}$ tests. Factors influencing MetS prevalence were identified by conducting multiple logistic regression analysis and examining odds ratios (ORs). All data analyses were performed using SPSS 24 version (IBM SPSS Statistics, Armonk, NY, USA).

\section{Results}

\subsection{General Characteristics}

The total number of study subjects was 578,416 . Of those, $243,222(42.0 \%)$ were male, and $335,194(58.0 \%)$ were female (Table 1$)$. Regarding age, there were $115,543(20.0 \%)$ subjects in the group of $40-49,229,769(39.7 \%)$ in the group of 50-59, 153,016 (26.5\%) in the group of 60-69, and 80,088 (13.8\%) in the group of 70 or older. Regarding income, 176,782 (30.6\%) were assigned to the fifth quintile group (the highest income level), which was the largest of the five quintile groups. Regarding location, $276,215(47.8 \%)$ lived in a metropolitan area, 230,053 (39.8\%) in a small- to medium-sized city, and $72,148(12.5 \%)$ in a farming/fishery rural area. With respect to health-related behaviors, $496,860(85.9 \%)$ were classified into the nonsmoking group and 81,556 (14.1\%) into the smoking group. Regarding drinking, 514,111 (88.9\%) were classified into the moderate drinking group and 64,305 (11.1\%) into the heavy drinking group. Lastly, 338,302 (58.5\%) were classified into the passive and 240,114 (41.5\%) in the active physical activity group. 
Table 1. General characteristics.

\begin{tabular}{|c|c|c|c|c|}
\hline \multicolumn{3}{|c|}{ Categories } & $n$ & $\%$ \\
\hline \multicolumn{3}{|c|}{ Total } & 578,416 & 100.0 \\
\hline \multirow{14}{*}{ Social Economic } & \multirow{2}{*}{ Sex } & Male & 243,222 & 42.0 \\
\hline & & Female & 335,194 & 58.0 \\
\hline & \multirow{4}{*}{ Age } & $40-49$ & 115,543 & 20.0 \\
\hline & & $50-59$ & 229,769 & 39.7 \\
\hline & & $60-69$ & 153,016 & 26.5 \\
\hline & & $\geq 70$ & 80,088 & 13.8 \\
\hline & \multirow{5}{*}{ Income } & 1st (the lowest) & 96,228 & 16.6 \\
\hline & & 2nd & 82,501 & 14.3 \\
\hline & & $3 r d$ & 97,137 & 16.8 \\
\hline & & 4 th & 125,768 & 21.7 \\
\hline & & 5th (the highest) & 176,782 & 30.6 \\
\hline & \multirow{3}{*}{ Location } & Metropolitan area & 276,215 & 47.8 \\
\hline & & Small- to medium-sized cities & 230,053 & 39.8 \\
\hline & & Farming/fishery rural & 72,148 & 12.5 \\
\hline \multirow{6}{*}{$\begin{array}{c}\text { Health-Related } \\
\text { Behavior }\end{array}$} & \multirow{2}{*}{ Smoking } & Nonsmoking & 496,860 & 85.9 \\
\hline & & Smoking & 81,556 & 14.1 \\
\hline & \multirow{2}{*}{ Drinking } & Moderate & 514,111 & 88.9 \\
\hline & & Heavy & 64,305 & 11.1 \\
\hline & \multirow{2}{*}{ Physical activity } & Passive & 338,302 & 58.5 \\
\hline & & Active & 240,114 & 41.5 \\
\hline
\end{tabular}

\subsection{Changes in Metabolic Syndrome Prevalence}

The prevalence of MetS increased from 28.5\% in 2011 and 28.6\% in 2013 to 31.7\% in 2015 (Table 2). The prevalence showed statistically significant differences according to sex, age, income, location, and health-related behaviors $(p<0.001)$. MetS prevalence was higher in men than in women, and increased with increasing age, with approximately $20 \%$ in the group of $40-49$ and over $40 \%$ in the group of 70 or older with a MetS diagnosis. Regarding income level, the prevalence was the lowest in the fifth quintile (the highest income level), and regarding location, it was the highest in farming/fishery rural areas and the lowest in metropolitan areas. With respect to health-related behaviors, the prevalence was higher in the smoking group, the heavy drinking group, and the passive group, compared to the nonsmoking group, the moderate drinking group, and the active group, respectively. The general characteristics according to the presence or absence of MetS was suggested as Supplemental Materials (Table S1).

Table 2. Changes of metabolic syndrome prevalence.

\begin{tabular}{|c|c|c|c|c|c|c|c|c|c|c|c|c|c|}
\hline \multirow{3}{*}{\multicolumn{2}{|c|}{ Categories }} & \multicolumn{4}{|c|}{2011} & \multicolumn{4}{|c|}{2013} & \multicolumn{4}{|c|}{2015} \\
\hline & & \multicolumn{2}{|c|}{ Normal } & \multicolumn{2}{|c|}{ MetS } & \multicolumn{2}{|c|}{ Normal } & \multicolumn{2}{|c|}{ MetS } & \multicolumn{2}{|c|}{ Normal } & \multicolumn{2}{|c|}{ MetS } \\
\hline & & $n$ & $\%$ & $n$ & $\%$ & $n$ & $\%$ & $n$ & $\%$ & $n$ & $\%$ & $n$ & $\%$ \\
\hline \multirow[b]{2}{*}{ Sex } & Male & 165,137 & 67.9 & 78,085 & 32.1 & 166,907 & 68.6 & 76,315 & 31.4 & 158,337 & 65.1 & 84,885 & 34.9 \\
\hline & Female & 248,343 & 74.1 & 86,851 & 25.9 & 246,172 & 73.4 & 89,022 & 26.6 & 236,630 & 70.6 & 98,564 & 29.4 \\
\hline \multirow{5}{*}{ Age } & $40-49$ & 167,810 & 80.9 & 39,596 & 19.1 & 127,035 & 81.6 & 28,642 & 18.4 & 90,919 & 78.7 & 24,624 & 21.3 \\
\hline & 50-59 & 144,630 & 71.2 & 58,398 & 28.8 & 164,291 & 73.4 & 59,517 & 26.6 & 165,057 & 71.8 & 64,712 & 28.2 \\
\hline & $60-69$ & 75,818 & 61.7 & 47,099 & 38.3 & 85,626 & 63.4 & 49,606 & 36.6 & 94,857 & 62.0 & 58,159 & 38.0 \\
\hline & $\geq 70$ & 25,222 & 56.0 & 19,843 & 44.0 & 36,127 & 56.8 & 27,572 & 43.2 & 44,134 & 55.1 & 35,954 & 44.9 \\
\hline & $\chi^{2}(p)$ & \multicolumn{4}{|c|}{$20160.172(<0.001)$} & \multicolumn{4}{|c|}{$19,524.344(<0.001)$} & \multicolumn{4}{|c|}{$16,332.791(<0.001)$} \\
\hline Income & 5 th & 125,767 & 72.6 & 47,541 & 27.4 & 127,177 & 72.3 & 48,742 & 27.7 & 121,865 & 68.9 & 54,917 & 31.1 \\
\hline
\end{tabular}


Table 2. Cont

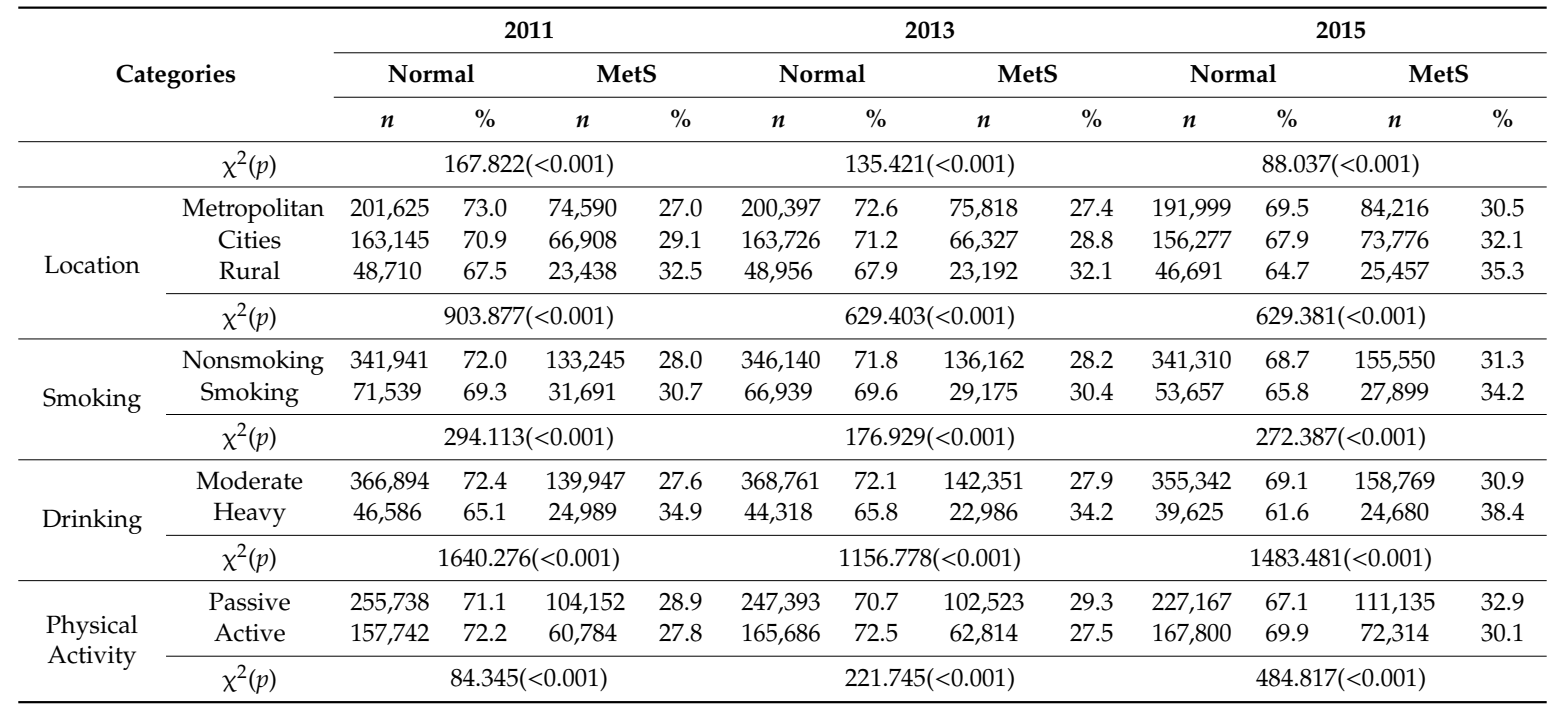

MetS: Metabolic Syndrome.

\subsection{Changes in Health-Related Behaviors}

Regarding health-related behavior changes, the largest group with respect to smoking was the continuous nonsmoking group $(457,133,79.0 \%)$, followed by the continuous smoking group $(63,012$, $10.9 \%)$, the short-term nonsmoking group $(39,727,6.9 \%)$, and the short-term smoking group $(18,544$, $3.2 \%)$. With respect to drinking, the largest group was the continuous moderate drinking group $(465,147,80.4 \%)$, followed by the short-term moderate group $(48,964,8.5 \%)$, the short-term heavy drinking group $(37,460,6.5 \%)$, and the continuous heavy drinking group $(26,845,4.6 \%)$. With respect to physical activity, the largest group was the continuous passive group $(183,138,31.7 \%)$, followed by the short-term active group $(161,729,28.0 \%)$, the short-term passive group $(155,164,26.8 \%)$, and the continuous active group $(78,385,13.5 \%)$.

There were differences in health-related behavior changes according to sex, age, income, and location $(p<0.001)$. Regarding sexual differences, $23.2 \%$ of men were classified into the continuous smoking group and the rate of women classified into the group was $2.0 \%$. Additionally, $9.8 \%$ of men and $0.9 \%$ of women were in the continuous heavy drinking group, and $30.3 \%$ of men and $32.7 \%$ of women were in the continuous passive group. Hence, with respect to health-related behavior changes, women engaged in healthier behaviors regarding smoking and drinking, whereas men were engaged in healthier physical activity. Regarding age differences, smoking and drinking decreased and physical activity increased with increasing age. Regarding differences by income level, the second and third quintile groups showed the highest rates of continuous smoking, heavy drinking, and passivity in physical activity. Regarding differences by location, the rate of active physical activity was higher in urban areas compared to rural areas, which also corresponded to the rates of smoking and drinking. (Table 3). The general characteristics according to health-related behavior was suggested as Supplemental Materials (Table S2). 
Table 3. Trend of changes in health-related behavior change by general characteristics.

\begin{tabular}{|c|c|c|c|c|c|c|c|c|c|c|}
\hline \multirow{2}{*}{\multicolumn{2}{|c|}{ Smoking }} & \multicolumn{2}{|c|}{$\begin{array}{l}\text { Continuous } \\
\text { Nonsmoking }\end{array}$} & \multicolumn{2}{|c|}{$\begin{array}{c}\text { Short-Term } \\
\text { Nonsmoking }\end{array}$} & \multicolumn{2}{|c|}{$\begin{array}{c}\text { Short-Term } \\
\text { Smoking }\end{array}$} & \multicolumn{2}{|c|}{$\begin{array}{l}\text { Continuous } \\
\text { Smoking }\end{array}$} & \multirow[t]{2}{*}{$\chi^{2}(p)$} \\
\hline & & $\mathbf{n}$ & $\%$ & $\mathbf{n}$ & $\%$ & n & $\%$ & $\mathbf{n}$ & $\%$ & \\
\hline \multicolumn{2}{|c|}{ Total } & 457,133 & 79.0 & 39,727 & 6.9 & 18,544 & 3.2 & 63,012 & 10.9 & \multirow{3}{*}{$\begin{array}{c}116,272.686 \\
(<0.001)\end{array}$} \\
\hline \multirow{2}{*}{ Sex } & Male & 140,397 & 57.7 & 31,925 & 13.1 & 14,444 & 5.9 & 56,456 & 23.2 & \\
\hline & Female & 316,736 & 94.5 & 7802 & 2.3 & 4100 & 1.2 & 6556 & 2.0 & \\
\hline \multirow{4}{*}{ Age } & $40-49$ & 83,155 & 72.0 & 8646 & 7.5 & 5069 & 4.4 & 18,673 & 16.2 & \multirow{4}{*}{$\begin{array}{c}10,819.038 \\
(<0.001)\end{array}$} \\
\hline & $50-59$ & 176,996 & 77.0 & 16,778 & 7.3 & 8171 & 3.6 & 27,824 & 12.1 & \\
\hline & $60-69$ & 126,100 & 82.4 & 10,222 & 6.7 & 3944 & 2.6 & 12,750 & 8.3 & \\
\hline & $\geq 70$ & 70,882 & 88.5 & 4081 & 5.1 & 1360 & 1.7 & 3765 & 4.7 & \\
\hline \multirow{5}{*}{ Income } & $1 \mathrm{st}$ & 75,379 & 78.3 & 6767 & 7.0 & 3311 & 3.4 & 10,771 & 11.2 & \multirow{5}{*}{$\begin{array}{l}2231.495 \\
(<0.001)\end{array}$} \\
\hline & 2nd & 62,150 & 75.3 & 6487 & 7.9 & 3062 & 3.7 & 10,802 & 13.1 & \\
\hline & $3 \mathrm{rd}$ & 74,379 & 76.6 & 7282 & 7.5 & 3427 & 3.5 & 12,049 & 12.4 & \\
\hline & 4th & 99,849 & 79.4 & 8763 & 7.0 & 3748 & 3.0 & 13,408 & 10.7 & \\
\hline & 5 th & 145,376 & 82.2 & 10,428 & 5.9 & 4996 & 2.8 & 15,982 & 9.0 & \\
\hline \multirow{3}{*}{ Location } & Metropolitan & 219,386 & 79.4 & 18,380 & 6.7 & 8662 & 3.1 & 29,787 & 10.8 & \multirow{3}{*}{$68.201(<0.001)$} \\
\hline & Cities & 181,009 & 78.7 & 16,096 & 7.0 & 7556 & 3.3 & 25,392 & 11.0 & \\
\hline & Rural & 56,738 & 78.6 & 5251 & 7.3 & 2326 & 3.2 & 7833 & 10.9 & \\
\hline \multirow{2}{*}{\multicolumn{2}{|c|}{ Drinking }} & \multicolumn{2}{|c|}{$\begin{array}{l}\text { Continuous } \\
\text { Moderate }\end{array}$} & \multicolumn{2}{|c|}{$\begin{array}{c}\text { Short-Term } \\
\text { Moderate }\end{array}$} & $\begin{array}{r}\text { Short } \\
\text { He }\end{array}$ & $\begin{array}{l}\text { erm } \\
y\end{array}$ & $\begin{array}{r}\text { Cont } \\
\mathrm{H}\end{array}$ & $\begin{array}{l}\text { lous } \\
y\end{array}$ & $2^{2}(n)$ \\
\hline & & $n$ & $\%$ & $n$ & $\%$ & $n$ & $\%$ & $n$ & $\%$ & \\
\hline & otal & 465,147 & 80.4 & 48,964 & 8.5 & 37,460 & 6.5 & 26,845 & 4.6 & \\
\hline & Male & 152,110 & 62.5 & 37,409 & 15.4 & 29,872 & 12.3 & 23,831 & 9.8 & $86,283.507$ \\
\hline sex & Female & 313,037 & 93.4 & 11,555 & 3.4 & 7588 & 2.3 & 3014 & 0.9 & $(<0.001)$ \\
\hline & $40-49$ & 83,584 & 72.3 & 11,722 & 10.1 & 11,048 & 9.6 & 9189 & 8.0 & \\
\hline Age & $50-59$ & 177,400 & 77.2 & 22,382 & 9.7 & 17,290 & 7.5 & 12,697 & 5.5 & $17,400.494$ \\
\hline Age & $60-69$ & 130,051 & 85.0 & 11,378 & 7.4 & 7341 & 4.8 & 4246 & 2.8 & $(<0.001)$ \\
\hline & $\geq 70$ & 74,112 & 92.5 & 3482 & 4.3 & 1781 & 2.2 & 713 & 0.9 & \\
\hline & $1 \mathrm{st}$ & 78,077 & 81.1 & 8120 & 8.4 & 5976 & 6.2 & 4055 & 4.2 & \\
\hline & $2 n d$ & 64,445 & 78.1 & 8022 & 9.7 & 5958 & 7.2 & 4076 & 4.9 & \\
\hline Income & 3rd & 76,675 & 78.9 & 9020 & 9.3 & 6,746 & 6.9 & 4696 & 4.8 & $\begin{array}{l}81 \% .730 \\
(<0.001)\end{array}$ \\
\hline & 4th & 101,285 & 80.5 & 10,622 & 8.4 & 8165 & 6.5 & 5696 & 4.5 & \\
\hline & 5 th & 144,665 & 81.8 & 13,180 & 7.5 & 10,615 & 6.0 & 8322 & 4.7 & \\
\hline & Metropolitan & 222,263 & 80.5 & 23,249 & 8.4 & 17,848 & 6.5 & 12,855 & 4.7 & \\
\hline Location & Cities & 185,039 & 80.4 & 19,386 & 8.4 & 14,965 & 6.5 & 10,663 & 4.6 & $10.592(<0.001)$ \\
\hline & Rural & 57,845 & 80.2 & 6329 & 8.8 & 4647 & 6.4 & 3327 & 4.6 & \\
\hline & & Conti & lous & Shor & & Short & erm & Cont & & \\
\hline Physi & al activity & Pas & ve & Pas & ve & Ac & $\%$ & Ac & \% & $\chi^{2}(p)$ \\
\hline & & $n$ & $\%$ & $n$ & $\%$ & $n$ & $\%$ & $n$ & $\%$ & \\
\hline & otal & 183,138 & 31.7 & 155,164 & 26.8 & 161,729 & 28.0 & 78,385 & 13.5 & \\
\hline & Male & 73,575 & 30.3 & 63,888 & 26.3 & 68,195 & 28.0 & 37,564 & 15.4 & 1423.333 \\
\hline Sex & Female & 109,563 & 32.7 & 91,276 & 27.2 & 93,534 & 27.9 & 40,821 & 12.2 & $(<0.001)$ \\
\hline & $40-49$ & 43,031 & 37.2 & 29,130 & 25.2 & 30,732 & 26.6 & 12,650 & 10.9 & \\
\hline Age & $50-59$ & 76,354 & 33.2 & 61,510 & 26.8 & 63,928 & 27.8 & 27,977 & 12.2 & 5346.539 \\
\hline Age & $60-69$ & 40,600 & 26.5 & 41,890 & 27.4 & 45,353 & 29.6 & 25,173 & 16.5 & $(<0.001)$ \\
\hline & $\geq 70$ & 23,153 & 28.9 & 22,634 & 28.3 & 21,716 & 27.1 & 12,585 & 15.7 & \\
\hline & $1 \mathrm{st}$ & 30,303 & 31.5 & 26,350 & 27.4 & 27,222 & 28.3 & 12,353 & 12.8 & \\
\hline & 2nd & 26,534 & 32.2 & 22,708 & 27.5 & 23,296 & 28.2 & 9963 & 12.1 & \\
\hline Income & $3 r d$ & 31,404 & 32.3 & 26,652 & 27.4 & 27,065 & 27.9 & 12,016 & 12.4 & $\begin{array}{l}882.334 \\
(<0.001)\end{array}$ \\
\hline & 4th & 40,038 & 31.8 & 33,663 & 26.8 & 35,277 & 28.0 & 16,790 & 13.3 & \\
\hline & 5 th & 54,859 & 31.0 & 45,791 & 25.9 & 48,869 & 27.6 & 27,263 & 15.4 & \\
\hline & Metropolitan & 79,963 & 28.9 & 74,004 & 26.8 & 79,786 & 28.9 & 42,462 & 15.4 & \\
\hline Location & Cities & 76,431 & 33.2 & 60,924 & 26.5 & 63,354 & 27.5 & 29,344 & 12.8 & $\begin{array}{l}3588.688 \\
(<0.001)\end{array}$ \\
\hline & Rural & 26,744 & 37.1 & 20,236 & 28.0 & 18,589 & 25.8 & 6579 & 9.1 & \\
\hline
\end{tabular}

\subsection{Health-Related Behavior Changes and Metabolic Syndrome Prevalence}

The examination of MetS prevalence according to health-related behavior changes showed that regarding smoking, the prevalence was the highest in the short-term nonsmoking group (37.2\%). Regarding drinking, the prevalence was the highest in the continuous heavy drinking group (40.8\%), and regarding physical activity, it was the highest in the short-term passive group (32.9\%) (Table 4). 
Of all health-related behavior groups, the continuous heavy drinking group showed the highest MetS prevalence $(40.8 \%)$ and the continuous active group the lowest prevalence $(29.4 \%)$.

Table 4. Health-related behavior change and metabolic syndrome prevalence.

\begin{tabular}{|c|c|c|c|c|c|c|c|c|}
\hline \multirow{2}{*}{\multicolumn{2}{|c|}{ Categories }} & \multicolumn{3}{|c|}{ Year } & \multicolumn{2}{|c|}{ Normal } & \multicolumn{2}{|c|}{ MetS } \\
\hline & & 2011 & 2013 & 2015 & $n$ & $\%$ & $n$ & $\%$ \\
\hline \multirow{9}{*}{ Smoking } & Nonsmoking & $x$ & $x$ & $x$ & 316,344 & 69.2 & 140,789 & 30.8 \\
\hline & \multirow{3}{*}{$\begin{array}{l}\text { Short-term } \\
\text { nonsmoking }\end{array}$} & 0 & $x$ & $x$ & \multirow{3}{*}{24,966} & \multirow{3}{*}{62.8} & \multirow{3}{*}{14,761} & \multirow{3}{*}{37.2} \\
\hline & & $\times$ & O & $x$ & & & & \\
\hline & & 0 & O & $x$ & & & & \\
\hline & \multirow{3}{*}{$\begin{array}{l}\text { Short-term } \\
\text { smoking }\end{array}$} & $\times$ & $x$ & 0 & \multirow{3}{*}{12,268} & \multirow{3}{*}{66.2} & \multirow{3}{*}{6,276} & \multirow{3}{*}{33.8} \\
\hline & & 0 & $x$ & 0 & & & & \\
\hline & & $\times$ & O & 0 & & & & \\
\hline & Smoking & 0 & O & 0 & 41,389 & 65.7 & 21,623 & 34.3 \\
\hline & \multicolumn{4}{|c|}{$\chi^{2}(p)$} & \multicolumn{4}{|c|}{$956.068(<0.001)$} \\
\hline \multirow{9}{*}{ Drinking } & Moderate & $x$ & $x$ & $x$ & 323,738 & 69.6 & 141,409 & 30.4 \\
\hline & \multirow{3}{*}{$\begin{array}{l}\text { Short-term } \\
\text { moderate }\end{array}$} & 0 & $x$ & $x$ & \multirow{3}{*}{31,604} & \multirow{3}{*}{64.5} & \multirow{3}{*}{17,360} & \multirow{3}{*}{35.5} \\
\hline & & $x$ & O & $x$ & & & & \\
\hline & & O & 0 & $x$ & & & & \\
\hline & \multirow{3}{*}{$\begin{array}{l}\text { Short-term } \\
\text { heavy }\end{array}$} & $x$ & $x$ & 0 & \multirow{3}{*}{23,724} & \multirow{3}{*}{63.3} & \multirow{3}{*}{13,736} & \multirow{3}{*}{36.7} \\
\hline & & 0 & $x$ & 0 & & & & \\
\hline & & $\times$ & O & 0 & & & & \\
\hline & Heavy drinking & 0 & 0 & 0 & 15,901 & 59.2 & 10,944 & 40.8 \\
\hline & \multicolumn{4}{|c|}{$\chi^{2}(p)$} & \multicolumn{4}{|c|}{$2127.236(<0.001)$} \\
\hline & Passive & $x$ & $x$ & $x$ & 123,011 & 67.2 & 60,127 & 32.8 \\
\hline & & 0 & $x$ & $x$ & & & & \\
\hline & passive & $x$ & O & $x$ & 104,156 & 67.1 & 51,008 & 32.9 \\
\hline & & O & O & $x$ & & & & \\
\hline Physical & & $x$ & $x$ & 0 & & & & \\
\hline & Snort-term & 0 & $x$ & 0 & 112,426 & 69.5 & 49,303 & 30.5 \\
\hline & & $\times$ & O & 0 & & & & \\
\hline & Active & 0 & O & 0 & 55,374 & 70.6 & 23,011 & 29.4 \\
\hline & & $\chi^{2}(p)$ & & & & 515.93 & $<0.001)$ & \\
\hline
\end{tabular}

$\times$ : Nonsmoking, moderated drinking, passive in physical activity; $\bigcirc$ : Smoking, heavy drinking, active in physical activity; MetS: Metabolic Syndrome.

\subsection{The Factors Influencing the Risk of Metabolic Syndrome Prevalence}

To identify factors influencing the risk of MetS prevalence, multiple logistic regression analysis was performed with the presence or absence of MetS as the dependent variable. The sex, area, age, income level, the status of smoking/drinking/activity, and the presence or absence of MetS were determined in the year 2015 data. Health-related behavior change was determined using data from 2011, 2013, and 2015. The analysis results are presented in Table 5. Two regression models were constructed with subjects' general characteristics controlled by including all the variables in each of the models. In Model 1, the current smoking, drinking, and physical activity status were added as independent variables, while in Model 2 the changes in smoking, drinking, and physical activity were included. The results of Model 1 showed that the risk for MetS was higher by $9.7 \%$ in the smoking group vs. the nonsmoking group, by $54.9 \%$ in the heavy drinking group vs. the moderate drinking group, and by $18.7 \%$ in the passive group vs. the active group. However, in Model 2, which included health-related behavior changes, it was found that the risk for MetS was the highest in the short-term nonsmoking group (by $22 \%$, using the continuous nonsmoking group as the reference), followed by the short-term smoking group (by 11.6\%) and the continuous smoking group (by 10.5\%). Regarding changes in drinking behavior, the risk for MetS increased from the short-term moderate group (by 32.3\%, with 
the continuous moderate drinking group as the reference) to the short-term heavy drinking group (by $48.3 \%$ ) to the heavy drinking group (by $84.9 \%$ ). Regarding changes in physical activity, the risk for MetS increased from the short-term active group (by $11.4 \%$, with the continuous active group as the reference) to the short-term passive group (by 23.9\%) to the continuous passive group (by $30.3 \%$ ). In addition to health-related behavior changes, the following characteristics affected the prevalence of MetS: it was higher in women than in men, in small- to medium-sized cities or in farming/fishery rural areas than in metropolitan areas, in age groups of 50 or older than in the age group of $40-49$, and in the fourth or lower-income quintile group than in the fifth quintile group.

Table 5. Multiple logistic regression for metabolic syndrome prevalence.

\begin{tabular}{|c|c|c|c|c|c|c|c|c|c|c|}
\hline \multirow{2}{*}{\multicolumn{2}{|c|}{ Variable }} & \multirow{3}{*}{$\begin{array}{c}\begin{array}{c}\text { Reference } \\
\text { Value }\end{array} \\
\text { Male }\end{array}$} & \multicolumn{4}{|c|}{ Model 1} & \multicolumn{4}{|c|}{ Model 2} \\
\hline & & & \multirow{2}{*}{$\begin{array}{c}\text { OR } \\
1.172\end{array}$} & \multicolumn{2}{|c|}{$95 \%$ CI } & \multirow{2}{*}{$\begin{array}{c}p \\
<0.001\end{array}$} & \multirow{2}{*}{$\begin{array}{c}\text { OR } \\
1.097\end{array}$} & \multicolumn{2}{|c|}{$95 \% \mathrm{CI}$} & \multirow{2}{*}{$\begin{array}{c}p \\
<0.001\end{array}$} \\
\hline Sex & Female & & & 1.157 & 1.187 & & & 1.082 & 1.111 & \\
\hline \multirow{2}{*}{ Area } & Farming/fishery rural area & \multirow{2}{*}{ Metropolitan } & 1.494 & 1.468 & 1.519 & $<0.001$ & 1.075 & 1.062 & 1.088 & $<0.001$ \\
\hline & Small- to medium-sized cities & & 2.444 & 2.401 & 2.488 & $<0.001$ & 1.112 & 1.092 & 1.132 & $<0.001$ \\
\hline \multirow{3}{*}{$\begin{array}{l}\text { Age } \\
\text { group }\end{array}$} & $50-59$ & \multirow[t]{3}{*}{$40-49$} & 3.345 & 3.278 & 3.414 & $<0.001$ & 1.505 & 1.480 & 1.531 & $<0.001$ \\
\hline & $60-69$ & & 1.103 & 1.084 & 1.122 & $<0.001$ & 2.511 & 2.466 & 2.556 & $<0.001$ \\
\hline & $\geq 70$ & & 1.096 & 1.076 & 1.117 & $<0.001$ & 3.481 & 3.410 & 3.553 & $<0.001$ \\
\hline \multirow{4}{*}{$\begin{array}{c}\text { Income } \\
\text { Level }\end{array}$} & 1st (the lowest) & \multirow{4}{*}{ 5th (the highest) } & 1.112 & 1.093 & 1.132 & $<0.001$ & 1.094 & 1.075 & 1.113 & $<0.001$ \\
\hline & 2nd & & 1.085 & 1.068 & 1.102 & $<0.001$ & 1.084 & 1.064 & 1.104 & $<0.001$ \\
\hline & $3 \mathrm{rd}$ & & 1.077 & 1.064 & 1.091 & $<0.001$ & 1.102 & 1.083 & 1.121 & $<0.001$ \\
\hline & 4 th & & 1.121 & 1.101 & 1.141 & $<0.001$ & 1.078 & 1.061 & 1.095 & $<0.001$ \\
\hline Smoking & Smoking & Nonsmoking & 1.097 & 1.078 & 1.116 & $<0.001$ & & & & \\
\hline Drinking & Heavy & Moderate & 1.549 & 1.521 & 1.579 & $<0.001$ & & & & \\
\hline Activity & Passive & Active & 1.187 & 1.173 & 1.200 & $<0.001$ & & & & \\
\hline \multirow{3}{*}{ Smoking } & Short-term nonsmoking & Nonsmoking & & & & & 1.220 & 1.192 & 1.248 & $<0.001$ \\
\hline & Short-term smoking & & & & & & 1.116 & 1.080 & 1.152 & $<0.001$ \\
\hline & Smoking & & & & & & 1.105 & 1.083 & 1.127 & $<0.001$ \\
\hline \multirow{3}{*}{ Drinking } & Short-term moderate & Moderate & & & & & 1.323 & 1.295 & 1.351 & $<0.001$ \\
\hline & Short-term heavy & & & & & & 1.483 & 1.449 & 1.518 & $<0.001$ \\
\hline & Heavy drinking & & & & & & 1.849 & 1.799 & 1.900 & $<0.001$ \\
\hline \multirow{3}{*}{$\begin{array}{l}\text { Physical } \\
\text { Activity }\end{array}$} & Passive & Active & & & & & 1.303 & 1.279 & 1.328 & $<0.001$ \\
\hline & Short-term passive & & & & & & 1.239 & 1.216 & 1.263 & $<0.001$ \\
\hline & Short-term active & & & & & & 1.114 & 1.093 & 1.135 & $<0.001$ \\
\hline
\end{tabular}

OR: odds ratio; $95 \%$ CI: 95\% confidence interval.

\section{Discussion}

In this descriptive longitudinal study, observational analysis was performed to identify factors influencing the prevalence of MetS, using data from the Korea National Health Examination Survey of a total of 578,416 adults aged 40 or older. Smoking, drinking, and physical activity are modifiable health-related behaviors and they are very critical in the management of MetS. Although MetS develops gradually and over a long time, most studies have examined whether subjects performed health-related behaviors only at the time of data collection, not considering the duration over which they performed such behaviors. To overcome this limitation, the present study utilized data from the Korea National Health Examination Survey conducted periodically (i.e., every other year) to examine the effects of health-related behavior changes. However, because health-related behaviors change over time, data from the Korea National Health Examination Survey in 2011, 2013, and 2015 were utilized to investigate the effects of health-related behavior changes on MetS prevalence.

\subsection{Metabolic Syndrome Prevalence}

The prevalence of MetS in Korea was 28.5\% in 2011, 28.6\% in 2013, and 31.7\% in 2015. According to the Korean Society of Cardiometabolic Syndrome, the $2015 \mathrm{MetS}$ prevalence was 20.3\% in adults aged 19 or older, $27.0 \%$ in adults aged 30 or older, and $37.7 \%$ in adults aged 65 or older [23]. Considering that this study's subjects were aged 40 or older, this study's findings on MetS prevalence appear 
representative. In a study based on the US National Health and Nutrition Examination Survey, MetS prevalence in 2014 in the US was also at a similar level (31.5\%), but the age range of this study sample was 20 to 85 [24]. In contrast, the sample of this study included adults aged 40 or older, and given that MetS prevalence increases with age, the prevalence in the population of the same age range as in the US study is likely to be lower in Korea. The discrepancy is attributable to the differences in abdominal obesity criteria (the NCEP-ATP III criterion of $80 \mathrm{~cm}$ vs. $85 \mathrm{~cm}$ in this study), genetic factors, and lifestyle habits [25].

MetS manifests differently depending on sex, age, and socioeconomic characteristics, and different studies have reported different findings. In this study, MetS prevalence was statistically significantly different according to sex, age, income, and location. The prevalence was higher in men than in women, and in older adults than in those who were younger [25]. These findings are congruous with the finding that abdominal obesity and blood pressure increase with aging. In addition, it is due to an increase in sedentary lifestyles, functional disabilities, and a decrease in physical activity with aging [26,27]. Regarding income, the highest quintile group showed the lowest prevalence, and regarding location, it was metropolitan areas that showed the lowest prevalence. These findings confirm an association between socioeconomic status and MetS prevalence, as shown by a previous study [26].

\subsection{Association between Health-Related Behavior Changes and Metabolic Syndrome Prevalence}

Health-related behaviors showed differences according to sex, age, income, and location. With respect to sex, women showed higher rates of continuous nonsmoking and moderate drinking. With respect to age, older age groups showed higher rates of continuous nonsmoking, moderate drinking, and physical activity. These findings suggest an association between maintaining healthy lifestyle habits and longevity. With respect to income, the rates of continuous smoking, heavy drinking, and passive physical activity were higher in the middle quintile groups (second through fourth) than in the lowest or highest quintile groups. This confirms the finding of various studies that a higher income is directly related to higher health status. Simultaneously, this suggests that the middle-income groups may neglect conducting a healthy lifestyle due to a lack of time, an information gap, and economic alienation, whereas the lowest income group is at the center of focus for various systems and can get assistance with healthy physical activity.

Different studies have reported different findings regarding the relationships between MetS and smoking, drinking, and physical activity. In some studies, the relationships were statistically significant while in others they were not. However, in this study MetS prevalence was higher in the smoking group than in the nonsmoking group, in the heavy drinking group than in the moderate drinking group, and in the passive group than in the active group, demonstrating statistically significant relationships between MetS and smoking, drinking, and physical activity [5,28].

A close examination of health-related behavior changes showed that the rates of groups attempting to quit smoking and drink moderately are higher compared with those who started to smoke or drink heavily, and that the rate of those who proactively tried to increase physical activity was also high. These findings are interpreted as indicating that the number of individuals highly interested in health-related behaviors and trying to maintain their health is increasing. Regarding the prevalence of MetS according to the changes in health-related behaviors, all groups, except for the continuous nonsmoking, continuous moderate drinking, and continuous active groups, showed MetS prevalence above the mean. These findings demonstrate the importance of considering not only the current status of smoking, drinking, and physical activity, but also health-related behaviors in the past and how long a health-related behavior has been maintained. In particular, there has been a close association demonstrated between smoking and MetS as well as its components [29], and it should be noted that in this study, MetS prevalence was higher in the short-term nonsmoking group than in the continuous smoking group. This finding urges researchers to consider why smokers try to quit smoking. Considering that they are motivated to quit smoking due to health reasons, the short-term 
nonsmoking group (i.e., those who smoked in the past but not currently) should undoubtedly be a subject of focus and managed consistently, in addition to the continuous smoking group.

\subsection{The Risk of Metabolic Syndrome Prevalence}

In the analysis based simply on the current smoking, drinking, and physical activity status as independent variables, the risk for MetS increased by $9.7 \%$ in the smoking group vs. the nonsmoking group, by $54.9 \%$ in the heavy drinking group vs. the moderate drinking group, and by $18.7 \%$ in the passive group vs. the active group. The greatest difference in the findings after health-related behavior changes were included in the analysis (Model 2) was that the risk of MetS prevalence was higher by $22 \%$ in the short-term nonsmoking group using the continuous nonsmoking group as the reference. In contrast, it was higher by $11.6 \%$ in the short-term smoking group and by $10.5 \%$ in the continuous smoking group, demonstrating that the risk is higher in the short-term nonsmoking group than it is in the continuous smoking group.

Numerous studies have reported that the risk for MetS increases in smokers compared to nonsmokers. In this study, however, the risk was found to be the highest in the group of former smokers who had currently quit smoking. Smokers who already had other health problems may have decided to quit smoking due to those health problems, which may have put them at an increased risk for MetS. An alternative interpretation of this finding is that weight gain occurred as a result of smoking cessation. The phenomenon of weight gain after smoking cession is well known. In comparison to those who continue smoking, weight, blood pressure, and cholesterol levels increase for individuals who quit smoking $[30,31]$. It has been argued that the health benefits due to smoking cessation may be lower than previously thought because weight gain elevates the risk for cardiovascular disease. However, a study reported that although weight initially increased with smoking cessation, the increase was attenuated over time, with no difference between former smokers and nonsmokers after five years [32]. Accordingly, more attention should be paid to the prevention of weight gain in the short-term nonsmoking group, and smokers attempting to quit smoking should be closely managed so that they do not return to smoking because of weight gain.

The risk for MetS increased by $84.9 \%$ in the continuous heavy drinking group compared with the continuous moderate drinking group, suggesting the need to avoid heavy drinking. The findings on the association between drinking alcohol and the prevalence of MetS and its components are inconsistent [33]. In this study, however, the risk for MetS associated with heavy drinking was higher than the risk associated with smoking or lack of physical activity, clearly showing the need for moderating drinking. In Korea, the rate of heavy drinking is very high and alcohol is the leading cause of the burden of disease [34]. Hence, an effort should be made to avoid heavy drinking to prevent MetS.

Regarding physical activity, MetS prevalence was higher in the passive groups than in the continuous active group, suggesting the need to increase physical activity. As the rate of physical activity is reported to be $38.7 \%$ in Korea while it is $50.3 \%$ in major OECD countries, this study's findings demonstrate the need to develop realistic and practical programs to increase physical activity.

Lastly, the risk for MetS was higher in small- to medium-sized cities and rural areas than in metropolitan areas, in adults aged 50 or older than those in their 40s, and in lower-income quintile groups than in the highest. It is speculated that location and income level affected MetS prevalence because geographical and economic factors made it difficult to develop interest in disease prevention, improve health-related behaviors, conduct a healthy lifestyle, and have access to health screening. Specifically, according to Model 2, persistent changes in health-related behaviors had the greatest impact in the risk of MetS prevalence, except for age, which is not modifiable. Therefore, the effect on the risk of MetS prevalence would be great if focus is consistently directed toward the improvement of health-related behaviors in adults between the ages of 40 and 49 with the second quintile of income, who, as shown in Table 3, continuously smoke, drink heavily, and are passive in their physical activity.

Therefore, using the results of this study, it can suggest which group is effective to focus on to reduce the MetS prevalence. In addition, the importance of health-related behavior has been proved 
through this study; thus, these findings can be used as a basic resource for policy decision making for the practice of a healthy lifestyle.

Our study has the following limitations. First, this study sample did not include adults younger than 40 years old. Second, various other data, such as the volume of food consumption and genetic factors, were not taken into consideration. Third, the survey of health-related behaviors was based on self-reports and thus the data may have been influenced by recall and social desirability biases, thereby reducing accuracy. Finally, since this study focused on MetS prevalence, we did not analyze how health-related behavior change had an impact on MS incidence. Despite these limitations, the present study had significant strengths. First, our large sample of 578,416 citizens aged 40 or older was representative of the Korean population in those age groups. Second, this study is the first of its kind to include changes in health-related behaviors in addition to the behaviors at the time of data collection. Future studies should identify factors affecting the prevalence of MetS by including a wider variety of independent variables and considering the duration and absolute amount of health-related behavior performance over a longer term. In addition, it is necessary to analyze whether health-related behavior change affects not only MetS prevalence but also MetS incidence.

\section{Conclusions}

This study was conducted to investigate the effect of health-related behavior change on the prevalence of MetS. Study findings showed that MetS prevalence was associated not only with socioeconomic factors but also with health-related behavior and change in the behavior. The prevalence increased in the smoking, heavy drinking, and passive groups compared with the nonsmoking, moderate drinking, and active groups, respectively. Regarding smoking, MetS prevalence was much higher in the short-term nonsmoking group (subjects who smoked in the past but not currently) than in the continuous smoking group. Accordingly, more attention should be paid to the health of smokers trying to quit smoking. In addition, the risk for MetS increased in heavy drinkers compared to moderate drinkers, suggesting the need to avoid heavy drinking. To summarize, the present study demonstrated that to prevent MetS, it is crucial to maintain healthy lifestyle habits.

Supplementary Materials: The following are available online at http://www.mdpi.com/2227-9032/8/2/134/s1, Table S1: The general characteristics according to the presence or absence of metabolic syndrome, Table S2: The general characteristics according to health-related behavior.

Author Contributions: Conceptualization, E.Y., K.L. and S.L.; formal analysis, I.P.; investigation, S.L.; methodology, E.Y., K.L. and I.P;; writing —original draft, E.Y. and K.L.; writing—review and editing, E.Y. and K.L. All authors have read and agreed to the published version of the manuscript.

Funding: This research received no external funding.

Conflicts of Interest: The authors declare no conflict of interest.

\section{References}

1. Pothiwala, P.; Jain, S.K.; Yaturu, S. Metabolic syndrome and cancer. Metab. Syndr. Relat. Disord. 2009, 7, 279-287. [CrossRef] [PubMed]

2. Sun, K.; Liu, J.; Ning, G. Active smoking and risk of metabolic syndrome: A meta-analysis of prospective studies. PLoS ONE 2012, 7, e47791. [CrossRef]

3. Alkerwi, A.; Boutsen, M.; Vaillant, M.; Barre, J.; Lair, M.L.; Albert, A.; Guillaume, M.; Dramaix, M. Alcohol consumption and the prevalence of metabolic syndrome: A meta-analysis of observational studies. Atherosclerosis 2009, 204, 624-635. [CrossRef]

4. Freiberg, M.S.; Cabral, H.J.; Heeren, T.C.; Vasan, R.S.; Ellison, R.C. Alcohol consumption and the prevalence of the metabolic syndrome in the U.S. A cross-sectional analysis of data from the Third National Health and Nutrition Examination Survey. Diabetes Care 2004, 27, 2954-2959. [CrossRef] [PubMed]

5. Santos, A.C.; Ebrahim, S.; Barros, H. Alcohol intake, smoking, sleeping hours, physical activity and the metabolic syndrome. Prev. Med. 2007, 44, 328-334. [CrossRef] [PubMed] 
6. Ford, E.S.; Kohl, H.W.; Mokdad, A.H.; Ajani, U.A. Sedentary behavior, physical activity, and the metabolic syndrome among U.S. adults. Obes. Res. 2005, 13, 608-614. [CrossRef] [PubMed]

7. Cleeman, J.I. Executive summary of the third report of the National Cholesterol Education Program (NCEP) expert panel on detection, evaluation, and treatment of high blood cholesterol in adults (adult treatment panel III). J. Am. Med. Assoc. 2001, 285, 2486-2497.

8. Yoo, S.; Kim, H.; Cho, H.I. Improvements in the metabolic syndrome and stages of change for lifestyle behaviors in korean older adults. Osong Public Heal. Res. Perspect. 2012, 3, 85-93. [CrossRef]

9. Eckel, R.H.; Grundy, S.M.; Zimmet, P.Z. The metabolic syndrome. Lancet 2005, 365, 1415-1428. [CrossRef]

10. Lim, S.; Shin, H.; Song, J.H.; Kwak, S.H.; Kang, S.M.; Yoon, J.W.; Choi, S.H.; Cho, S.I.; Park, K.S.; Lee, H.K.; et al. Increasing prevalence of metabolic syndrome in Korea: The Korean National Health and Nutrition Examination Survey for 1998-2007. Diabetes Care 2011, 34, 1323-1328. [CrossRef]

11. Shin, H.Y.; Lee, J.Y.; Kim, J.E.; Lee, S.; Youn, H.; Kim, H.; Lee, J.; Park, M.S.; Huh, S. Cause-of-death statistics in the Republic of Korea, 2014. J. Korean Med. Assoc. 2016, 59, 221-232. [CrossRef]

12. Jackson, S.E.; West, R.; Brown, J. If at first you don't succeed, when should you try again? A prospective study of failed quit attempts and subsequent smoking cessation. Addict. Behav. 2020, 106, 106366. [CrossRef] [PubMed]

13. Kwasnicka, D.; Dombrowski, S.U.; White, M.; Sniehotta, F.F. 'It's not a diet, it's a lifestyle': A longitudinal, data-prompted interview study of weight loss maintenance. Psychol. Health 2019, 34, 963-982. [CrossRef]

14. Nanri, A.; Tomita, K.; Matsushita, Y.; Ichikawa, F.; Yamamoto, M.; Nagafuchi, Y.; Kakumoto, Y.; Mizoue, T. Effect of six months lifestyle intervention in Japanese men with metabolic syndrome: Randomized controlled trial. J. Occup. Health 2012, 54, 215-222. [CrossRef]

15. Bo, S.; Ciccone, G.; Baldi, C.; Benini, L.; Dusio, F.; Forastiere, G.; Lucia, C.; Nuti, C.; Durazzo, M.; Cassader, M.; et al. Effectiveness of a lifestyle intervention on metabolic syndrome. A randomized controlled trial. J. Gen. Intern. Med. 2007, 22, 1695-1703. [CrossRef]

16. Oh, E.G.; Bang, S.Y.; Hyun, S.S.; Kim, S.H.; Chu, S.H.; Jeon, J.Y.; Im, J.A.; Lee, M.K.; Lee, J.E. Effects of a 6-month lifestyle modification intervention on the cardiometabolic risk factors and health-related qualities of life in women with metabolic syndrome. Metabolism 2010, 59, 1035-1043. [CrossRef]

17. Christian, J.G.; Byers, T.E.; Christian, K.K.; Goldstein, M.G.; Bock, B.C.; Prioreschi, B.; Bessesen, D.H. A computer support program that helps clinicians provide patients with metabolic syndrome tailored counseling to promote weight loss. J. Am. Diet. Assoc. 2011, 111, 75-83. [CrossRef]

18. Schoenborn, C.A.; Adams, P.F.; Peregoy, J.A. Health behaviors of adults: United States, 2008-2010. Vital Health Stat. 2013, 10, 1-184.

19. Cho, S.M.J.; Lee, H.; Pyun, W.B.; Kim, H.C. Differential control rate of systolic and diastolic blood pressure among Korean adults with hypertension: The Sixth Korean national health and nutrition examination survey, 2013-2015 (KNHANES VI). Korean Circ. J. 2019, 49, 1035-1048. [CrossRef]

20. Bull, F.C.; Maslin, T.S.; Armstrong, T. Global physical activity questionnaire (GPAQ): Nine country reliability and validity study. J. Phys. Act. Health 2009, 6, 790-804. [CrossRef]

21. Grundy, S.M.; Cleeman, J.I.; Daniels, S.R.; Donato, K.A.; Eckel, R.H.; Franklin, B.A.; Gordon, D.J.; Krauss, R.M.; Savage, P.J.; Smith, S.C.; et al. Diagnosis and management of the metabolic syndrome: An American Heart Association/National Heart, Lung, and Blood Institute scientific statement. Circulation 2005, 112, 2735-2752. [CrossRef] [PubMed]

22. Kim, M.K.; Lee, W.Y.; Kang, J.H.; Kang, J.H.; Kim, B.T.; Kim, S.M.; Kim, E.M.; Suh, S.H.; Shin, H.J.; Lee, K.R.; et al. 2014 clinical practice guidelines for overweight and obesity in Korea. Endocrinol. Metab. 2014, 29, 405-409. [CrossRef] [PubMed]

23. Huh, J.H.; Lee, J.H.; Moon, J.S.; Sung, K.C.; Kim, J.Y.; Kang, D.R. Metabolic Syndrome Severity Score in Korean Adults: Analysis of the 2010-2015 Korea National Health and Nutrition Examination Survey. J. Korean Med. Sci. 2019, 34, e48. [CrossRef]

24. Marcotte-Chénard, A.; Deshayes, T.A.; Ghachem, A.; Brochu, M. Prevalence of the metabolic syndrome between 1999 and 2014 in the United States adult population and the impact of the 2007-2008 recession: An NHANES study. Appl. Physiol. Nutr. Metab. 2019, 44, 861-868. [CrossRef]

25. Oh, J.Y.; Hong, Y.S.; Sung, Y.A.; Barrett-Connor, E. Prevalence and factor analysis of metabolic syndrome in an urban Korean population. Diabetes Care 2004, 27, 2027-2032. [CrossRef] [PubMed] 
26. Moore, J.X.; Chaudhary, N.; Akinyemiju, T. Metabolic syndrome prevalence by race/ ethnicity and sex in the united states, national health and nutrition examination survey, 1988-2012. Prev. Chronic Dis. 2017, 14, 1-16. [CrossRef]

27. Mankowski, R.T.; Aubertin-leheudre, M.; Beavers, D.P.; Buford, T.W.; Church, T.; Glynn, N.W.; King, A.C.; Liu, C.; Manini, T.M.; Marsh, A.P.; et al. Sedentary time is associated with the metabolic syndrome in older adults with mobility limitations-The LIFE Study. Exp. Gerontol. 2015, 70, 32-36. [CrossRef]

28. Yoon, Y.S.; Oh, S.W.; Baik, H.W.; Park, H.S.; Kim, W.Y. Alcohol consumption and the metabolic syndrome in Korean adults: The 1998 Korean National Health and Nutrition Examination Survey. Am. J. Clin. Nutr. 2004, 80, 217-224. [CrossRef]

29. Park, S.H. Smoking-related differential influence of alcohol consumption on the metabolic syndrome. Subst. Use Misuse 2019, 54, 2351-2358. [CrossRef]

30. Chinn, S.; Jarvis, D.; Melotti, R.; Luczynska, C.; Ackermann-Liebrich, U.; Antó, J.M.; Cerveri, I.; De Marco, R.; Gislason, T.; Heinrich, J.; et al. Smoking cessation, lung function, and weight gain: A follow-up study. Lancet 2005, 365, 1629-1635. [CrossRef]

31. Yoon, C.; Goh, E.; Park, S.M.; Cho, B. Effects of smoking cessation and weight gain on cardiovascular disease risk factors in Asian male population. Atherosclerosis 2010, 208, 275-279. [CrossRef] [PubMed]

32. Reas, D.L.; Nygård, J.F.; Sørensen, T. Do quitters have anything to lose? Changes in body mass index for daily, never, and former smokers over an 11-year period (1990-2001). Scand. J. Public Health 2009, 37, 774-777. [CrossRef] [PubMed]

33. Suliga, E.; Kozieł, D.; Ciesla, E.; Rebak, D.; Głuszek-Osuch, M.; Gluzek, S. Consumption of alcoholic beverages and the prevalence of metabolic syndrome and its components. Nutrients 2019, 11, 2764. [CrossRef]

34. Lim, S.S.; Vos, T.; Flaxman, A.D.; Danaei, G.; Shibuya, K.; Adair-Rohani, H.; Amann, M.; Anderson, H.R.; Andrews, K.G.; Aryee, M.; et al. A comparative risk assessment of burden of disease and injury attributable to 67 risk factors and risk factor clusters in 21 regions, 1990-2010: A systematic analysis for the Global Burden of Disease Study 2010. Lancet 2012, 380, 2224-2260. [CrossRef]

(C) 2020 by the authors. Licensee MDPI, Basel, Switzerland. This article is an open access article distributed under the terms and conditions of the Creative Commons Attribution (CC BY) license (http://creativecommons.org/licenses/by/4.0/). 